\title{
A Search for Radio-loud Supernovae
}

\author{
Elaine M. Sadler and Duncan Campbell-Wilson \\ School of Physics, University of Sydney, NSW 2006, Australia \\ ems@physics.usyd.edu.au \\ Received 1996 December 12, accepted 1997 February 14
}

\begin{abstract}
Some extragalactic supernovae, such as SN 1986J in NGC 891 (Rupen et al. 1987), are unusually strong radio sources. Their radio emission typically peaks a few years after the supernova explodes, and appears to arise from the interaction of the supernova shock with a dense stellar wind shed by the progenitor star. Since two radio-loud and possibly optically faint supernovae have recently been found serendipitously in nearby spiral galaxies, it is possible that such objects are common. If so, this would have important consequences for our understanding of both the chemical enrichment history of galaxies and the radio emission from starburst galaxies. Preliminary results from a survey of nearby spiral galaxies with the Molonglo Observatory Synthesis Telescope (MOST) allow us to make a first estimate of the radio supernova rate. A larger study now in progress will provide a much more sensitive measurement.
\end{abstract}

Keywords: Supernovae: general — surveys — radio sources: variable — radio sources: galaxies

\section{What are Radio-loud Supernovae?}

Several supernovae have now been discovered serendipitously as strong radio sources even though they were not seen optically at the time of explosion. SN 1986 J in NGC 891 (Rupen et al. 1987) and SN 1978K in NGC 1313 (Ryder et al. 1993) are two examples. The progenitors of these radio-loud supernovae appear to be massive $\left(>30 \mathrm{M}_{\odot}\right)$ red giants in which a high rate of mass loss continued up to the moment of explosion. The radio emission arises from interaction of the supernova shock wave with this dense stellar wind.

It is important to distinguish between radio supernovae and classical supernova remnants. The emission from radio supernovae arises in material directly surrounding the star, and peaks between a few months and a few years after explosion (after which it declines slowly for several decades). Radio supernovae can be far more powerful radio sources than Galactic supernova remnants (SN 1986J at its peak was over a thousand times more powerful than Cas A).

A more systematic investigation of radio-loud supernovae (RSNe) is likely to be important for several reasons:

- If some radio-loud supernovae are optically dim or invisible (as SN 1986J appears to have been), and if these are also the most massive stars (with a corresponding large yield of processed stellar material), then there are important consequences for our understanding of the chemical enrichment history of galaxies.
- Because of their high radio luminosities and slow decay times, RSNe may be significant contributors to the total radio flux of starburst galaxies, and hence of interest in studying the FIR-radio correlation.

- Long-term monitoring of RSNe at several frequencies may yield valuable clues to the circumstellar environment of massive stars.

- If an RSN is detected sufficiently early, VLBI measurements of the expanding shell offer the possibility of a direct distance determination, and hence a measurement of the Hubble constant (e.g. Bartel 1988).

\section{Radio Spectra and 'Light Curves'}

Chevalier $(1982,1984)$ presented a model for the radio emission from Type II (and Type Ib) supernovae, in which the rapidly-moving supernova ejecta interacts with a slow-moving wind from pre-supernova mass loss. Such a model can reproduce much of the observed late-time evolution of the radio flux density and spectral index [though recent results for SN 1993J (van Dyk et al. 1994) show that the Chevalier model does not adequately describe the early behaviour of radio light curves for this supernova, perhaps due to variations in the mass-loss rate and clumping in the stellar wind, which are not yet accounted for in the model].

The radio emission usually peaks later, and is stronger, at lower frequencies (Weiler et al. 1986), so most $\mathrm{RSNe}$ have steep radio spectra at late times 
and are most easily detected at frequencies below $1 \mathrm{GHz}$.

With two or more radio images of a galaxy taken at different epochs, it is possible to search for RSNe using a template subtraction method analogous to the 'plate blinking' method used in optical supernova searches. The main difference (Figure 1) is that the optical decay time is only a few months, while the radio decay time is many years. An optical supernova search requires that each target field be observed at least every few weeks to be sure of detecting any supernova that appears. The much longer decay time in the radio, however, means that observations a few years apart are all that is needed for complete coverage. For example, Ryder et al. (1993) were able to get a reasonable radio 'light curve' spanning 10 years for SN $1978 \mathrm{~K}$ from two archival images of NGC 1313 taken in 1982 and 1986, plus a third observation made in 1992.

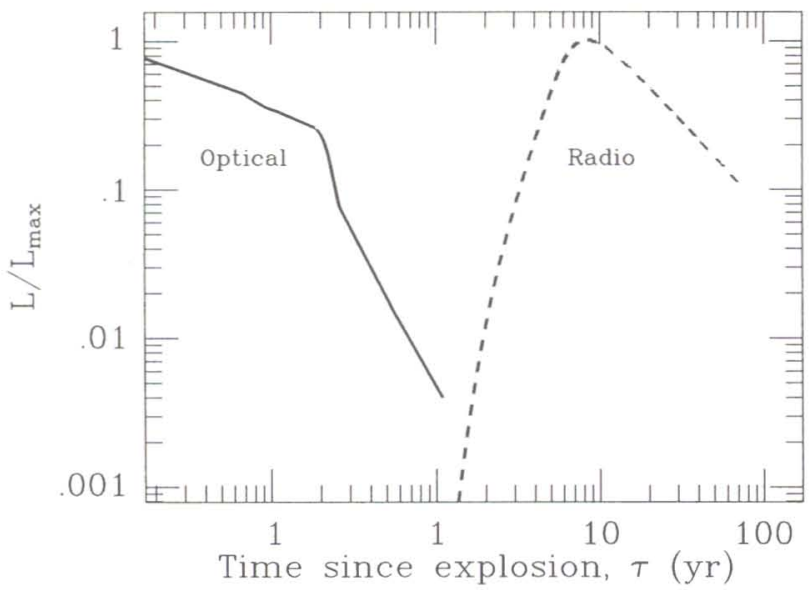

Figure 1-A comparison of the expected optical (B) and radio $(843 \mathrm{MHz})$ light curves for a radio supernova. Here $L / L_{\max }$ is the ratio of the observed luminosity to that at maximum. The optical line shows the mean type IIP supernova light curve tabulated by Doggett \& Branch (1985), while the radio light curve is based on the model calculated for SN 1986J by Weiler et al. (1996).

\section{A Radio Supernova Search with the MOST}

Archival data from the Molonglo Observatory Synthesis Telescope (MOST) now span more than a decade (1982-96), and represent a valuable resource for studies of time-varying radio emission. The telescope's operating frequency of $843 \mathrm{MHz}$ is well suited to observing RSNe, and the fixed format and resolution make it easy to compare archival data with new observations. To investigate the feasibility of a large-scale MOST search for radio supernovae, we carried out a pilot study in late 1992/93.

Our targets were nearby (heliocentric radial velocity $v_{\mathrm{h}}<2000 \mathrm{~km} \mathrm{~s}^{-1}$ ), spiral (type Sa or later) galaxies from the Revised Shapley-Ames Catalog (RSA; Sandage \& Tammann 1981) which had already been observed with MOST at least twice during the period 1982-92. The 12 galaxies which met these criteria were re-observed with MOST during 1992-93. We excluded NGC 1313, the parent galaxy of SN 1978K, on the possibly dubious grounds that it was known a priori to contain the kind of object we were looking for. Six of the 12 galaxies have had one or more recent optical supernovae.

To test for the presence of time-varying radio emission in MOST maps, we would ideally like to perform a complete subtraction of the two maps. In our pilot study, however, the pairs of maps available for comparison often had slightly different field centres, which made proper subtraction difficult with the existing reduction software. As an alternative, one of us (DCW) made a list of the positions and flux densities of all sources in each pair of MOST maps being studied (to date, we have only compared the 1992/93 map with the most recent archive map). We then compared the two lists and noted any significant changes.

We estimate that we can detect the following:

- a 'new' source (i.e. one that is present in the later map but not the earlier one) at a level of $10 \mathrm{mJy}$ or above;

- changes in source brightness of $20 \%$ or more for a source seen in two maps (for sources of $10 \mathrm{mJy}$ and above);

- the disappearance (due to fading) of 'an 'old' source that is present in an earlier map at a level of $10 \mathrm{mJy}$ or above, but has fallen below the detection limit in the later map.

While these numbers are probably conservative, they give us a benchmark for estimating the radio supernova rate. A further restriction is that a new source close to a strong galaxy core may be hard to recognise. In principle, therefore, sensitivity to new sources may be lower within about 1 arcmin of the nucleus.

\section{A First Estimate of the Radio Supernova Rate}

We found no new sources in any of the 12 galaxies during the pilot study. One galaxy (NGC 4945) was dominated by a very strong ( $\sim 5 \mathrm{Jy})$ unresolved nuclear source. 'Spikes' and other artifacts from this source mean that the much weaker underlying extended emission is poorly mapped, and a new source would probably need to be of at least $15 \mathrm{mJy}$ to be recognised, so we excluded this galaxy from the statistical study described below.

In calculating supernova rates, we follow the method outlined for optical supernovae by van den Bergh, McClure \& Evans (1987) and Evans, van den Bergh \& McClure (1989). The aim is to determine, for each galaxy observed, the 'control time' during which we would have been able to detect a given event. Evans et al. also defined the 'surveillance time' as the control time (in years) divided by the 
galaxy's optical luminosity (in units of $10^{10} \mathrm{~L}_{\mathrm{B}, \odot}$ ). The number of detected supernovae divided by the surveillance time then gives the supernova rate per unit stellar luminosity. Since radio supernovae clearly cover a range in radio luminosity, we calculated the probability of detecting two well observed radio-loud supernovae, SN 1986J and the less luminous SN 1978K.

The control time is calculated from model radio light curves (i.e. flux density as a function of time) for SN 1978K and SN 1986J, scaled to the distance of each target galaxy. Figure 2 shows the model light curves adopted. For SN 1986J, the light curve was calculated using the model parameters derived by Weiler et al. (1996; their Table 2) and shifted to the MOST observing frequency of $843 \mathrm{MHz}$ using the calculated radio spectral index $\alpha$. For SN $1978 \mathrm{~K}$, the curve is from Ryder et al. (1993), fitted to the early $843 \mathrm{MHz}$ observations and calculated as described in their Figure 4.

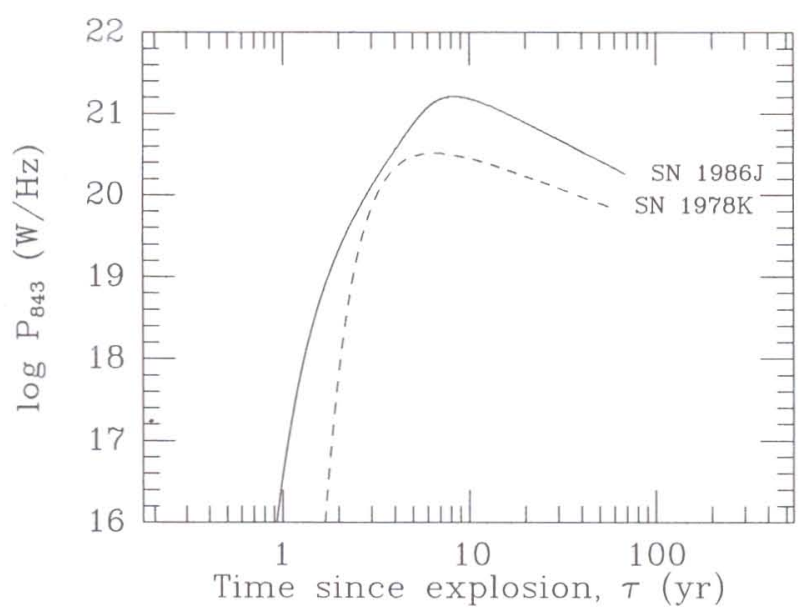

Figure 2-Model radio light curves for SN 1986J and SN $1978 \mathrm{~K}$ at $843 \mathrm{MHz}$, assuming $H_{0}=75 \mathrm{~km} \mathrm{~s}^{-1} \mathrm{Mpc}^{-1}$.

The 'surveillance time' is simply the control time scaled by the (blue) galaxy luminosity (galaxies vary in size, so this lets us calculate the rate per fixed number of stars rather than per galaxy). As an example, we show the calculation of 'control time' and 'surveillance time' for NGC 1097:

\section{- SN 1978K}

The peak $843 \mathrm{MHz}$ flux density for SN $1978 \mathrm{~K}$ scaled to the distance of NGC 1097 is predicted to be $9.5 \mathrm{mJy}$. Thus an RSN like SN $1978 \mathrm{~K}$ will always lie below our $10 \mathrm{mJy}$ detection limit, and so the control time is zero no matter how long the galaxy is observed for.

- SN 1986J

The peak $843 \mathrm{MHz}$ flux density for SN 1986J scaled to the distance of NGC 1097 is predicted to be $46 \cdot 4 \mathrm{mJy}$. The model light curve implies that the flux density first rises above $10 \mathrm{mJy}$ on day 1450 (i.e. $4 \cdot 0$ years) after the SN explodes, and remains above $10 \mathrm{mJy}$ until day 14470 (i.e. $39 \cdot 6$ years after the explosion). In principle, therefore, an RSN like SN 1986J should be detectable for a total of 35.6 years before fading below the detection limit.

An additional constraint, however, comes from our assumption that the RSN must vary in brightness by at least $20 \%$ between the two observations for it to be recognised as variable. The slow rate of decline at late times means that this becomes difficult unless there is a long time between the two epochs. For NGC 1097, where the observations are separated by $4 \cdot 2$ years, we can detect an RSN as variable if it exploded no more than 15.5 years before the first-epoch observation (rather than the $39 \cdot 6$ years implied by the detection limit alone). Figure 3 shows the relation between the interval between observations $(\Delta T)$ and the range of 'time since explosion' $(\tau)$ for which an RSN can be detected.

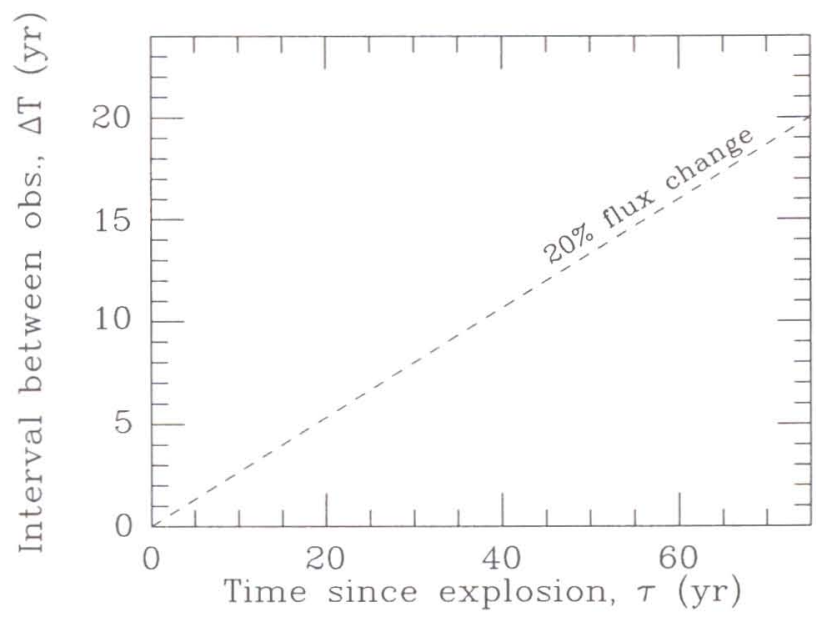

Figure 3-Time span $\Delta T$ needed to detect an RSN on the declining part of its light curve (assuming that the observed flux density must vary by $20 \%$ or more for the object to be recognised as variable), as a function of the elapsed time $\tau$ between the supernova explosion and the first observation. Note the large gain if archival observations span a long interval-each extra year added to $\Delta T$ adds roughly $3 \cdot 8$ years to $\tau$,

Note that the control time is independent of the value assumed for $H_{0}$, while the surveillance time, and hence the derived supernova rate, depends on $H_{0}$ only in the sense that the optical luminosity calculated for a galaxy (and hence the number of stars it is assumed to contain) depends on the precise distance adopted.

Table 1 lists the control time and surveillance time for galaxies in the pilot study. The total (blue) optical luminosity $L_{\mathrm{B}}$ is also listed for each galaxy, along with the time interval $\Delta T$ between the two radio observations. Here, we adopt $H_{0}=100 h \mathrm{~km} \mathrm{~s}^{-1} \mathrm{Mpc}^{-1}$.

For objects like SN $1978 \mathrm{~K}$, we have a total surveillance time of $62 \cdot 4 h^{-2}$ years and have detected 
Table 1. Calculation of 'surveillance time' for galaxies in the pilot study

\begin{tabular}{lcccccc}
\hline Galaxy & $\begin{array}{c}L_{\mathrm{B}} \\
\left(10^{10} \mathrm{~L}_{\odot}\right)\end{array}$ & $\begin{array}{c}\Delta T \\
(\mathrm{yr})\end{array}$ & $\begin{array}{c}\text { Control time } \\
(\mathrm{yr})\end{array}$ & \multicolumn{2}{c}{$\begin{array}{c}\text { Surveillance time } \\
\left(\mathrm{yr} / 10^{10} \mathrm{~L}_{\odot}\right)\end{array}$} \\
& & & $1978 \mathrm{~K}$ & $1986 \mathrm{~J}$ & $1978 \mathrm{~K}$ & $1986 \mathrm{~J}$ \\
\hline NGC 1097 & $3 \cdot 2 h^{-2}$ & $4 \cdot 2$ & 0 & $15 \cdot 7$ & 0 & $51 \cdot 0 h^{-2}$ \\
NGC 1365 & $5 \cdot 9 h^{-2}$ & $4 \cdot 1$ & 0 & $15 \cdot 1$ & 0 & $89 \cdot 1 h^{-2}$ \\
NGC 1433 & $1 \cdot 1 h^{-2}$ & $6 \cdot 9$ & $15 \cdot 0$ & $29 \cdot 6$ & $16 \cdot 9 h^{-2}$ & $33 \cdot 2 h^{-2}$ \\
NGC 1448 & $1 \cdot 1 h^{-2}$ & $4 \cdot 1$ & $9 \cdot 7$ & $16 \cdot 1$ & $10 \cdot 8 h^{-2}$ & $17 \cdot 9 h^{-2}$ \\
NGC 1532 & $1 \cdot 3 h^{-2}$ & $4 \cdot 1$ & $7 \cdot 1$ & $16 \cdot 0$ & $9 \cdot 4 h^{-2}$ & $21 \cdot 2 h^{-2}$ \\
NGC 1566 & $3 \cdot 2 h^{-2}$ & $4 \cdot 2$ & 0 & $15 \cdot 7$ & 0 & $50 \cdot 3 h^{-2}$ \\
NGC 1792 & $1 \cdot 4 h^{-2}$ & $4 \cdot 0$ & $9 \cdot 0$ & $15 \cdot 4$ & $12 \cdot 6 h^{-2}$ & $21 \cdot 6 h^{-2}$ \\
NGC 5236 & $1 \cdot 1 h^{-2}$ & $2 \cdot 8$ & $8 \cdot 5$ & $9 \cdot 1$ & $9 \cdot 3 h^{-2}$ & $10 \cdot 0 h^{-2}$ \\
NGC 7064 & $0 \cdot 1 h^{-2}$ & $7 \cdot 1$ & $30 \cdot 3$ & $30 \cdot 7$ & $3 \cdot 4 h^{-2}$ & $3 \cdot 5 h^{-2}$ \\
NGC 7213 & $2 \cdot 4 h^{-2}$ & $7 \cdot 1$ & 0 & $19 \cdot 3$ & 0 & $45 \cdot 7 h^{-2}$ \\
NGC 7590 & $0 \cdot 8 h^{-2}$ & $6 \cdot 0$ & 0 & $22 \cdot 9$ & 0 & $18 \cdot 3 h^{-2}$ \\
& Total surveillance time $\left(\mathrm{yr} / 10^{10} \mathrm{~L}_{\mathrm{B}, \odot)}\right.$ & & $62 \cdot 4$ & $361 \cdot 3$ \\
\hline
\end{tabular}

Table 2. Comparison of optical and radio supernova rates

\begin{tabular}{|c|c|c|c|c|c|}
\hline & \multicolumn{2}{|c|}{$\begin{array}{c}\text { Radio SN rate } \\
\left(\mathrm{SNe} / 100 \mathrm{yr} / 10^{10} \mathrm{~L} \odot\right)\end{array}$} & \multicolumn{3}{|c|}{$\begin{array}{c}\text { Optical SN rate } \\
\left(\mathrm{SNe} / 100 \mathrm{yr} / 10^{10} \mathrm{~L}_{\odot}\right)\end{array}$} \\
\hline & $1978 \mathrm{~K}$-like & 1986J-like & $\mathrm{SN} \mathrm{Ia}$ & SN Ib & SN II \\
\hline $\mathrm{Sa}$ & $\ldots$ & $<2 \cdot 2 h^{2}$ & $0.6 h^{2}$ & & 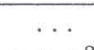 \\
\hline $\mathrm{Sab}, \mathrm{Sb}$ & $<3.8 h^{2}$ & $<0.7 h^{2}$ & $0 \cdot 3 h^{2}$ & $0.6 h^{2}$ & $1 \cdot 8 h^{2}$ \\
\hline Sbc-Sd & $<2 \cdot 8 h^{2}$ & $<0.6 h^{2}$ & $0 \cdot 2 h^{2}$ & $0 \cdot 4 h^{2}$ & $1 \cdot 3 h^{2}$ \\
\hline All Sa-Sd & $<1 \cdot 6 h^{2}$ & $<0 \cdot 28 h^{2}$ & $0 \cdot 3 h^{2}$ & $0.4 h^{2}$ & $1 \cdot 3 h^{2}$ \\
\hline
\end{tabular}

$<1$ RSN. Our detection rate for spiral galaxies $\left(\mathrm{Sa}^{-}\right.$ $\mathrm{Sd}$ ) is therefore $<1.6 h^{2}$ objects/century $/ 10^{10} \mathrm{~L}_{\mathrm{B}, \odot \text {. }}$. For objects like SN 1986J, we have a total surveillance time of $361 \cdot 3 h^{-2}$ years, and have again detected $<1$ RSN. This gives a detection rate for spiral galaxies of $<0 \cdot 28 h^{2}$ objects/century $/ 10^{10} \mathrm{~L}_{\mathrm{B}, \odot}$.

Table 2 compares the radio supernova limits derived here with the optical supernova rates derived by Evans et al. (1989). For objects like SN 1978K, our limits are not yet very restrictive - we cannot rule out a picture in which almost all Type II and Type Ib supernovae behave like SN 1978K. For more powerful RSNe like SN 1986J, we can already say that they probably represent no more than $25 \%$ of all Type II supernovae (and no more than 18\% of Types Ib and II combined), so they must be reasonably rare objects.

It is interesting to compare the total surveillance times for radio and optical SNe in our study and those of Evans et al. (1989) respectively. Evans et al. totals $3416 h^{-2}$ years $/ 10^{10} \mathrm{~L}_{\mathrm{B}, \odot}$ for Type II SNe (and a similar time for Type $\mathrm{Ib} \mathrm{SNe}$, which are also believed to come from massive stars) based on regular observations of 855 target galaxies over an eight-year period.

We have already reached a total of $361 h^{-2}$ years $/ 10^{10} \mathrm{~L}_{\mathrm{B}, \odot}$ for $1986 \mathrm{~J}-$ like objects by observing only 11 targets twice each with an interval of 4-5 years between observations. Thus it should be possible to reach a total surveillance time that approaches or exceeds that of the Evans et al. optical search by making only a modest number of new radio observations. Since the objects we are seeking are presumably rarer than optical $\mathrm{SNe}$, it is also clear that we need a total surveillance time at least this long to gather reasonable statistics.

\section{Optical Supernovae in the Pilot Galaxies}

Since several supernovae have been optically identified in our pilot galaxies in the past decade, we can also use these to estimate the fractional radio supernova rate. Table 3 lists the optical supernovae (Barbon, Cappellaro \& Turatto 1989; van den Bergh 1996) that would have been detectable by the pilot study if they were radio sources.

Table 3. Optical supernovae which could have been detected in the pilot study

\begin{tabular}{cccc}
\hline Galaxy & At $1978 \mathrm{~K}$ level & At $1986 \mathrm{~K}$ level & SN type? \\
\hline NGC 1097 & None & None & \\
NGC 1365 & None & $1983 \mathrm{~V}$ & Ib \\
NGC 1433 & $1985 \mathrm{P}$ & $1985 \mathrm{P}$ & II \\
NGC 1448 & $1983 \mathrm{~S}$ & $1983 \mathrm{~S}$ & II \\
NGC 1532 & $1981 \mathrm{~A}$ & $1981 \mathrm{~A}$ & II \\
NGC 1566 & None & None & \\
NGC 1792 & None & None & \\
NGC 5236 & $1983 \mathrm{~N}$ & $1983 \mathrm{~N}$ & Ib \\
NGC 7064 & None & None & \\
NGC 7213 & None & None & \\
NGC 7590 & None & None & \\
\hline
\end{tabular}

Five optical SNe (2 Type Ib, 3 Type II) could have been detected as radio sources at the 1986J level, and four (1 Type Ib, 3 Type II) at the 1978K level. This suggests (with large uncertainty because of the small number of optical detections) that RSNe like 1986J represent no more than $30 \%$ 
of Type II SNe (and no more than $20 \%$ of Types Ib and II combined), in agreement with the rates derived earlier. It also suggests (again with large uncertainty) that RSNe like $1978 \mathrm{~K}$ represent no more than $25 \%$ of Types Ib and II SNe combined, which is somewhat more restrictive than our earlier result.

\section{A Five-year Survey of Southern Spiral Galaxies with MOST}

Following on from the pilot study, we have now begun a five-year program to search a larger sample of galaxies for radio supernovae. To have the best chance of detecting $\mathrm{RSNe}$, and hence determining their true frequency, we restrict the search to galaxies that are sufficiently nearby that most RSNe should be detectable above our nominal MOST detection limit of $10 \mathrm{mJy}$. We therefore chose a subset of the RSA galaxy sample (also monitored by Evans et al.), using the following criteria:

- The galaxy is in the RSA Catalog.

- It is a spiral or irregular, i.e. type Sa or later (in general, E and S0 galaxies produce only Type Ia $\mathrm{SNe}$, whereas all the known RSNe appear to be Type II or Type Ib SNe).

- It is nearby $\left(v_{h}<1400 \mathrm{~km} \mathrm{~s}^{-1}\right)$.

- It lies south of $-25^{\circ}$ declination (set by the declination limit of the MOST).

- The LMC and SMC are excluded because they are too large for us to cover effectively.

This gives us 81 target galaxies, of which 75 are also in the Evans et al. (1989) sample of RSA galaxies monitored optically and 33 have already been observed at least once with the MOST (9 of them in our pilot study). We aim to observe each of these galaxies at least twice over the five-year period 1993-98.

What kind of limits should this study set on the radio supernova rate? Scaling from the pilot study, we should reach total surveillance times of at least $2500 h^{-2}$ years $/ 10^{10} \mathrm{~L}_{\mathrm{B}, \odot}$ for objects like $1986 \mathrm{~J}$ and $625 h^{-2}$ years $/ 10^{10} \mathrm{~L}_{\mathrm{B}, \odot}$ for objects like $1978 \mathrm{~K}$.
If no RSNe were actually detected, our upper limit for the RSN rate would be roughly $0.04 h^{2}$ /century/10 ${ }^{10} \mathrm{~L}_{\mathrm{B}, \odot}$ for objects like 1986J, and $0 \cdot 15 h^{2}$ /century/10 ${ }^{10} \mathrm{~L}_{\mathrm{B}, \odot}$ for objects like $1978 \mathrm{~K}$. This would allow us to say that no more than $3 \%$ and $12 \%$ respectively of Type II SNe are RSNe at the levels of $1986 \mathrm{~J}$ and $1978 \mathrm{~K}$.

It is likely that the detection limits adopted for the pilot study (10 mJy and $20 \%$ variation in flux density) are over-cautious, and that careful subtraction would allow us to reach detection limits of perhaps 2-3 mJy. In this case, the surveillance time associated with each observation would of course be correspondingly higher.

A byproduct of this search will be a uniform set of radio images of southern spiral galaxies, most of which have not previously been mapped by any radio telescope.

Barbon, R., Cappellaro, E., \& Turatto, M. 1989, A\&AS, 81,421

Bartel, N. 1988, in IAU Symposium 129, The Impact of VLBI on Astrophysics and Geophysics, p. 175

Chevalier, R. A. 1982, ApJ, 259, 302

Chevalier, R. A. 1984, ApJ, 285, L63.

Doggett, J. B., \& Branch, D. 1985, AJ, 90, 2303

Evans, R., van den Bergh, S., \& McClure, R. D. 1989, ApJ, 345,752

Rupen, M. P., van Gorkom, J. H., Knapp, G. R., Gunn, J. E., \& Schneider, D. P. 1987, AJ, 94, 61

Ryder, S., et al. 1993, ApJ, 416, 167

Sandage, A., \& Tammann, G. A. 1981, A Revised ShapleyAmes Catalog of Bright Galaxies (Washington: Carnegie Institute)

van den Bergh, S. 1996, in IAU Colloquium 145: Supernovae and Supernova Remnants, p. 1

van den Bergh, S., McClure, R. D., \& Evans, R. 1987, ApJ, 323,44

van Dyk, S. D., Weiler, K. W., Sramek, R. A., Rupen, M. P. \& Panagia, N. 1994, ApJ Lett., 432, L115

Weiler, K. W., Sramek, R. A., Panagia, N., van der Hulst, J. M., \& Salvati, M. 1986, ApJ, 301, 790

Weiler, K. W., van Dyk, S. D., Sramek, R. A., \& Panagia, N. 1996, in IAU Colloquium 145: Supernovae and Supernova Remnants, p. 283 